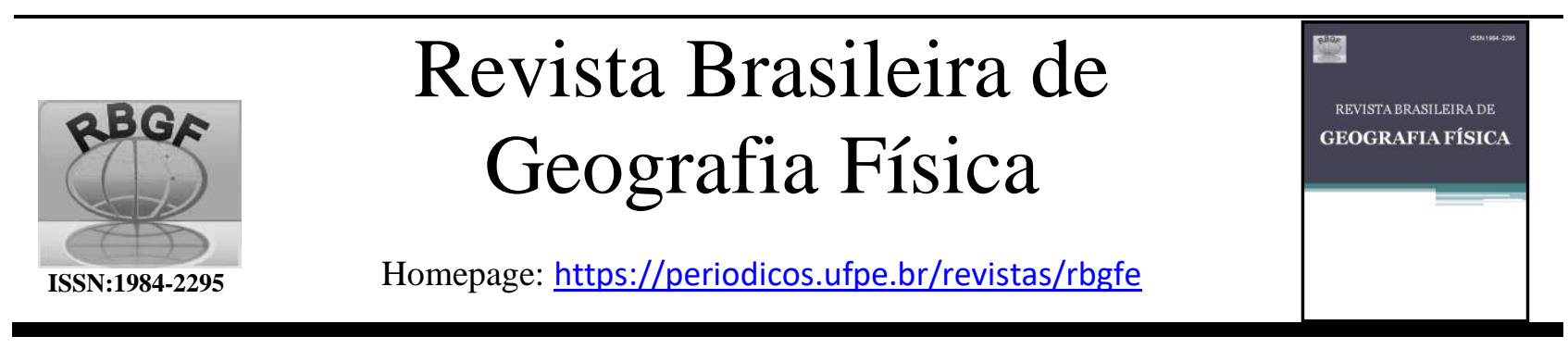

\title{
Atributos Físicos e Estoque de Carbono em Sistemas Agroflorestais nos Cerrados do Oeste da Bahia
}

\author{
Vandayse Abades Rosa ${ }^{1}$; Joaquim Pedro Soares Neto ${ }^{2}$ Heliab Bomfim Nunes ${ }^{2}$; Klever de Sousa Calisto ${ }^{3}$; \\ Joice das Neves Cruz ${ }^{3}$;
}

${ }^{1}$ Engenheira Agrônoma, Universidade do Estado da Bahia; Barreiras, BA, deyseabades@hotmail.com (autor para correspondência); ${ }^{2}$ Professor Doutor da Universidade do Estado da Bahia, Campus IX, Barreiras, BA, jpsneto@uneb.br; ${ }^{3}$ Bonsista de Iniciação científica da Universidade do Estado da Bahia, Campus IX, Barreiras, BA.

Artigo recebido em 15/04/2019 e aceito em 11/11/2019

\section{R E S U M O}

Os sistemas agroflorestais (SAF's) têm permitido a recuperação de áreas degradadas, a produção de alimentos, madeiras e fibras, além de gerar serviços ambientais, como aumento da matéria orgânica do solo e conservação da biodiversidade. Sendo assim, objetivou-se avaliar os efeitos dos SAF's nos atributos físicos do solo e no estoque de carbono em áreas de cerrado do oeste da Bahia. Os sistemas avaliados foram: Cerrado nativo, sistema com cacau/banana/arvores nativas com 18 anos de uso (SAF.1), eucalipto/pastagem com 8 anos de uso (SAF.2) e eucalipto de rebrota/pastagem (SAF.3). As amostras foram coletadas em camadas de $0,0-0,10 ; 0,10-0,20 ; 0,20-0,30 ; 0,30-0,40$ e $0,40-0,50$ m em quatro repetições. A análise de variância foi processada de acordo como delineamento inteiramente casualizado, além disso, realizou-se uma análise de componentes principais com o objetivo de verificar quais parâmetros poderiam diferenciar. Os sistemas apresentaram alterações na densidade do solo, diâmetro médio ponderado e diâmetro médio geométrico dos agregados, agregados maiores que $2 \mathrm{~mm}$, agregados menores que $2 \mathrm{~mm}$ e estoque de carbono orgânico. Na camada superficial todos os sistemas apresentaram Ds superior ao da área de CN. Os índices DMP, DMG, agregados maiores que 2 mm e estoque de carbono foram alterados pelos SAF's, principalmente nas camadas até $0,30 \mathrm{~m}$. De acordo com a análise de componentes principais tanto os atributos físicos como o carbono apresentaram altas correlações com a componente principal 1 e apenas o carbono com alta correlação com a componente principal 2.

Palavras chave: Qualidade do solo, sequestro de $\mathrm{CO}_{2}$, matéria orgânica.

\section{Physical Attributes and Carbon Stock in Agroforestry Systems in the "Cerrados" of Western Bahia}

\begin{abstract}
A B S T R A C T
Agroforestry systems (SAFs) have allowed the recovery of degraded areas, the production of food, wood and fibers crops, as well as generating environmental services such as increased soil organic matter and biodiversity conservation. The aim of this work was to evaluate the effects of SAFs on soil physical attributes and carbon stock in a QuartzarenicOrthicNeosol of the "Cerrados" of western Bahia.The evaluated aspects were the native "Cerrado", system with cacao / banana / native trees with 18 years of use (SAF.1), eucalyptus / pasture with 8 years of use (SAF.2) and eucalyptus of regrowth / pasture (SAF.3).Samples were collected in layers of 0.0-0.10; 0.10-0.20; 0.20-0.30; 0.30-0.40 and $0.40-0.50 \mathrm{~m}$ in four replicates in each plot and analysis of variance processed according to a completely randomized design.In addition, an analysis of main components was carried out in order to verify which parameters could differentiate or indicate similarity between the systems. The systems presented changes in soil density, weighted mean diameter, geometric mean diameter, aggregates larger than $2 \mathrm{~mm}$, aggregates smaller than $2 \mathrm{~mm}$ and organic carbon stock.In the surface layer, all the systems presented DS higher than of that of CN. DMP, DMG, aggregates larger than $2 \mathrm{~mm}$ and carbon stock were altered by SAF's, mainly in layers up to $0.30 \mathrm{~m}$. Principal component analysis showed that both physical attributes and carbon had high correlations with the main component 1 and only the carbon with high correlation with the main component 2.
\end{abstract}

Key words: Soil quality, CO2 sequestration, organic matter.

Rosa; V. A., Soares Neto J.P.S., Nunes; H.B., Calisto; K. S., Cruz J. N. 


\section{Introdução}

A conversão de agrossistemas naturais, para agricultura em monocultivo na região do cerrado tem sido amplamente discutida nos últimos anos, visto que, práticas convencionais de preparo do solo mal utilizadas, tem provocado significativa redução nos teores de carbono orgânico, com alterações nos principais elementos químicos $\mathrm{e}$ degradando o solo (Loss et al., 2014; Freitas et al., 2018), o que por sua vez provoca a emissão de gases para atmosfera (Fracetto et al., 2015), resultando em desequilíbrio no efeito estufa natural (Pulrolnik, 2009).

Atualmente, buscam-se práticas que visam à mitigação do aquecimento global, sem perder de vista a produção de alimentos e o desenvolvimento sustentável, como, por exemplo, o estabelecimento de sistemas de produção eficazes para o sequestro e o armazenamento de $\mathrm{C}$ na biomassa e no solo (Silva, 2013). Nesse sentido, um campo que atualmente vem sendo bastante explorado como pratica sustentável é o uso dos Sistemas Agroflorestais (SAFs).

Os Sistemas Agroflorestais (SAF) são sistemas de uso da terra onde espécies florestais e não florestais são cultivadas simultaneamente com cultivos anuais ou perenes (Shi et al., 2018). Esses sistemas de cultivo possuem vantagens socioeconômicas e benefícios ambientais tais como: recuperação de áreas degradadas, a produção diversificada de cultivos (alimentares, madeireiros e medicinais), como aumento de matéria orgânica no solo (Rocha Junior et al., 2017), conservação da biodiversidade, constituindo importantes sistemas de sequestro de carbono $\left(\mathrm{CO}_{2}\right)$ da atmosfera (Torralba, 2016).

Entre os sistemas de uso da terra, os Sistemas Agroflorestais são aqueles que acumulam o maior ativo de biomassa devido a intercalação de duas ou mais espécies o que, consequentemente, possuem o maior potencial de estoque de carbono (Linhares et al., 2016), além disso, matem as propriedades químicas intrínsecas do solo, eletronegatividade dos coloides organominerais e baixo PCZ (Barbosa et al., 2016). Em Sistemas Agroflorestais, o componente arbóreo, além de contribuir com a diminuição da erosão e da perda de água, ajuda na ciclagem de nutrientes e na manutenção de matéria orgânica, melhorando a estrutura e a qualidade do solo (Assis et al., 2015, Linhares et al., 2016).

Portanto, é importante que se desenvolvam mais pesquisas relacionadas ao potencial de sequestro e ao armazenamento de carbono em Sistemas Agroflorestais, pois esses, além serem um instrumento importante para mitigar os problemas do efeito estufa (Rocha, 2014) surge também como uma opção estratégica para produtores, graças à diversificação da produção e rentabilidade.

Nesse sentido objetivou-se com esse trabalho avaliar a influência de Sistemas Agroflorestais sobre os atributos físicos do solo e o estoque de carbono orgânico na região Oeste da Bahia.

\section{Material e métodos}

\section{Caracterização da Área em Estudo}

$\mathrm{O}$ estudo foi realizado na fazenda planalto (coordenadas: $12^{\circ} 08^{\prime} 38,6^{\prime \prime}$ S e $44^{\circ} 55^{\prime} 45,8^{\prime \prime}$ W) e no sitio jacarandá (coordenadas: $12^{\circ} 05^{\prime} 39,0^{\prime \prime}$ ' $\mathrm{S}$ e 445'21,2" W) situadas no município de Barreiras na Região Oeste da Bahia. No sítio jacarandá foi selecionado um talhão de 1 hectare com 18 anos de cultivo, denominado SAF.1 cultivado com cacau/banana/árvores nativas. $\mathrm{Na}$ fazenda planalto foram selecionados dois talhões, denominados: SAF.2 - cultivado com eucalipto/pastagem com idade de 8 anos e SAF.3também com eucalipto/pastagem o qual recebeu corte e foi conduzido a rebrota, além de uma área de cerrado nativo $(\mathrm{CN})$.

\section{Clima e Solo}

De acordo a classificação de Koppen o clima predominante da região é do tipo Aw, ou seja, tropical sub úmido com período chuvoso de outubro a abril e período seco de maio a setembro. O solo das áreas avaliadas foi classificado como Neossolo Quartzarênico órtico (Cardoso et al., 2009) e o resultados das analises granulométricas das áreas encontram-se na Tabela1. 
Tabela 1. Análise granulométrica das áreas em estudo, Barreiras-BA, 2018.

\begin{tabular}{|c|c|c|c|c|}
\hline \multirow[t]{2}{*}{ Sistema } & \multirow[t]{2}{*}{ Camada (m) } & \multicolumn{3}{|c|}{ Granulometria (g.kg ${ }^{-1}$ ) } \\
\hline & & Areia & Silte & Argila \\
\hline \multirow[t]{5}{*}{$\mathrm{CN}$} & $0-0,10$ & 889,00 & 34,86 & 76,14 \\
\hline & $0,10-0,20$ & 868,50 & 49,90 & 81,60 \\
\hline & $0,20-0,30$ & 888,95 & 36,82 & 74,23 \\
\hline & $0,30-0,40$ & 855,56 & 62,30 & 82,14 \\
\hline & $0,40-0,50$ & 850,08 & 68,55 & 81,37 \\
\hline \multirow[t]{5}{*}{ SAF.1 } & $0-0,10$ & 878,15 & 45,97 & 75,88 \\
\hline & $0,10-0,20$ & 862,05 & 42,07 & 95,88 \\
\hline & $0,20-0,30$ & 844,13 & 59,01 & 96,86 \\
\hline & $0,30-0,40$ & 814,37 & 73,53 & 112,10 \\
\hline & $0,40-0,50$ & 769,5 & 100,46 & 130,02 \\
\hline \multirow[t]{5}{*}{ SAF.2 } & $0-0,10$ & 871,55 & 25,46 & 102,98 \\
\hline & $0,10-0,20$ & 868,43 & 29,85 & 101,73 \\
\hline & $0,20-0,30$ & 871,87 & 21,27 & 106,86 \\
\hline & $0,30-0,40$ & 850,47 & 32,48 & 117,06 \\
\hline & $0,40-0,50$ & 839,09 & 40,07 & 120,84 \\
\hline \multirow[t]{5}{*}{ SAF.3 } & $0-0,10$ & 865,26 & 52,49 & 82,55 \\
\hline & $0,10-0,20$ & 862,05 & 42,07 & 95,88 \\
\hline & $0,20-0,30$ & 844,13 & 59,01 & 96,86 \\
\hline & $0,30-0,40$ & 214,37 & 73,53 & 112,10 \\
\hline & $0,40-0,50$ & 814,11 & 77,91 & 107,97 \\
\hline
\end{tabular}




\section{Amostragem}

Foram abertos minitricheiras em quatro pontos nos talhões selecionados nas profundidades de $0-0,10 ; 0,10-0,20 ; 0,20-0,30 ; 0,30-0,40$ e $0,40-0,50 \mathrm{~m}$, nos quais foram coletadas amostras indeformadas com o auxilio de um trado e anel volumétrico para as análises de densidade e estabilidade de agregados. E amostras deformadas para as analise granulométrica e carbono orgânico total.

As amostras foram devidamente identificadas e encaminhadas para o laboratório de Física dos Solos da Universidade do Estado da Bahia (UNEB), Campus IX para realização das análises.

\section{Analise granulométrica}

Esta foi determinada pelo método da pipeta por dispersão total utilizando como agente dispersante do hidróxido de sódio $(\mathrm{NaOH})$ de acordo com a metodologia da (Embrapa, 2011).

\section{Densidade do Solo}

A densidade do solo foi determinada pelo método do anel volumétrico segundo (Embrapa, 2011), coletou-se amostras de solo indeformadas em duplicata, totalizando 40 repetições, em anéis tipo Kopeckye. No laboratório foi pesado o conjunto (anel+solo) e anotou-se o valor. O conjunto foi levado à estufa de circulação forçada à105 ${ }^{\circ} \mathrm{C}$ até peso constante. A densidade foi calculada segundo a equação 1 :

$$
\mathrm{Ds}=(\mathbf{M s} / \mathrm{V})
$$

Onde: Ms é massa do solo seca à $105{ }^{\circ} \mathrm{C}$ e V é o volume do anel.

\section{Estabilidade de agregados em água}

A estabilidade dos agregados foi determinada em amostras destorroadas cuidadosamente com as mãos quebrando os agregados maiores em seus pontos de fragilidade natural e posteriormente secas ao ar. Após esse processo, as amostras foram passadas num conjunto de peneiras, os agregados retidos na peneira de $8-10 \mathrm{~mm}$ foram utilizados para serem processados. Retirou-se 4 amostras de $50 \mathrm{~g}$ por cada profundidade, em seguida essas amostras foram colocadas na parte superior de um jogo de peneiras de malhas 2,00 mm; 1,00 mm;0,50 mm;0,25 mm e $0,106 \mathrm{~mm}$ (Figura 3b). Estas foram umedecidas com um atomizador e aguardado 10 minutos. Em seguida as amostras foram imersas no tanque do aparelho de Yoder para oscilação vertical por 15 minutos. Os agregados retidos em cada peneira foram transferidos para cápsulas de alumínio e levadas a estufa de circulação forçada por 24 horas a $105{ }^{\circ} \mathrm{C}$ e na sequencia foi pesada cada fração (Salton, 2012).

Diâmetro médio ponderado: calculado a partir da expressão 2 (Coutinho, 2010).

$$
\mathbf{D M P}=\mathrm{S} \text { xiyi }
$$

em que: $\mathbf{i}=$ intervalo de classe: $2,0=X>1,0 \mathrm{~mm}$, de $1,0=X>0,5 \mathrm{~mm}$, de $0,5=X>0,25 \mathrm{~mm}$ e de $0,25=\mathrm{X}>0,105 \mathrm{~mm} ; \mathbf{x i}=$ é o diâmetrodo centro de classe $(\mathrm{mm}) ; \mathbf{y i}=$ é a razão entrea massa de agregados dentro da classe (xi) e amassa total de agregados.

Diâmetro médio geométrico: calculado a partir da expressão 3 (Coutinho, 2010).

$$
\text { DMG = expSwiln xi / ( S wi) }
$$

Em que: wi= peso dos agregados de cada centro de classe $(\mathrm{g})$; $\mathbf{l n}=$ logaritmo natural de $\mathrm{xi} ; \mathbf{x i}=$ diâmetrodo centro de classe ( $\mathrm{mm})$.

\section{Carbono orgânico total}

O carbono orgânico total, foi determinado por oxidação a quente com dicromato de potássio em meio sulfúrico (Embrapa, 2011) e o estoque de carbono em cada uma das camadas amostradas foi estimado a partir da expressão 4:

$$
\mathbf{E s t C}=\mathrm{C} \times \text { Ds } \mathrm{x} \text { e, }
$$

Onde: EstC é o estoque de carbono em $\mathrm{Mg}^{-1}{ }^{-1}$; $\mathbf{C}$ indica o teor de carbono orgânico em \%; Ds é densidade do solo da camada estudada em g.cm ${ }^{-3}$ e "e" a espessura da camada em centímetros (ALMEIDA,2014).

\section{Correção dos estoques de carbono pela massa equivalente}

Os estoques totais em cada camada foram corrigidos pelo método da massa equivalente segundo Carvalho et al. (2009) através da expressão 5:

$$
\mathrm{EstC}=\mathrm{Cs} * \mathrm{Ds} *((\mathrm{Dref} / \mathrm{Ds}) * \mathrm{e}) / 10 \text {. }
$$

Onde: $\mathbf{E s t C}=$ estoque de $\mathrm{C}$ orgânico em determinada profundidade $\left(\mathrm{Mg}_{\mathrm{h}} \mathrm{ha}^{-1}\right), \mathbf{C s}=$ teor de $\mathrm{C}$ orgânico total na profundidade amostrada ( $\mathrm{g} . \mathrm{kg}$ 1), Ds= densidade aparente do solo na profundidade amostrada $\left(\mathrm{kg}_{\mathrm{dm}} \mathrm{dm}^{-3}\right)$, Dref $=$ densidade do solo para profundidade amostrada na área de referência $\left(\mathrm{kg} \cdot \mathrm{dm}^{-3}\right), \mathbf{e}=$ espessura da camada considerada (cm). 


\section{CO2-Equivalente}

O dióxido de carbono seqüestrado foi calculado pela equação 6: (3,67 é a relação entre a massa molecular de $\mathrm{C}$ e $\mathrm{CO}_{2}$ ).

\section{$\mathbf{C O}_{2}=3,67 *$ Estoque de carbono ......(6) Análise estatística}

Os dados foram submetidos ao teste $\mathrm{F}$ da análise de variância a 5\% de probabilidade de erro e, se significativos foi aplicado o teste de média de Scoot-Knott através do programa estatístico SISVAR 5.6. (Ferreira, 2006). Esses dados também foram submetidos análises de componentes principais (ACP) com o objetivo de verificar quais os parâmetros físicos e o carbono orgânico poderiam ser utilizados para diferenciar ou para indicar similaridades entre os sistemas de agroflorestas. Essa análise foi processada no software PAST versão 2.17c (Hammer et al, 2001).
Resultados e discussão

$\mathrm{Na}$ Tabela 2 encontram-se os valores médios de densidade do solo nas áreas estudadas. Observa-se que na camada $0,00-0,10$ e $0,10-0,20 \mathrm{~m}$ a área de cerrado nativo apresentou a menor densidade, enquanto que entre as demais áreas não foi verificada diferenças estatísticas. Para as demais camadas não houve diferenças estatísticas entre as áreas. Esse valores de densidade segundo Silva et al. (2015) apresentam se acima do valor critico podendo acarretar restrições ao crescimento radicular como também infiltração de água no solo. Por outro lado, as características físicas do solo não estão relacionadas apenas com a forma de uso do solo, a textura do mesmo influencia de forma direta, portanto, esses valores da DS podem ser devido ao caráter arenoso da área estudada.

Tabela 2. Densidade do solo em áreas cultivadas com sistemas agroflorestais na região Oeste da Bahia, Barreiras, 2018.

\begin{tabular}{|c|c|c|c|c|c|}
\hline \multirow[t]{2}{*}{ Sistema } & \multicolumn{5}{|c|}{ Camada $(\mathbf{m})$} \\
\hline & $\begin{array}{c}0- \\
0,10\end{array}$ & $\begin{array}{c}0,10- \\
0,20\end{array}$ & $\begin{array}{c}0,20- \\
0,30\end{array}$ & $\begin{array}{c}0,30- \\
0,40\end{array}$ & $, 40-0,50$ \\
\hline & & 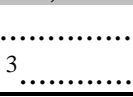 & & $\ldots \ldots \ldots \ldots . . . . K$ & g.dm ${ }^{-}$ \\
\hline $\mathbf{C N}$ & $1,47 \mathrm{aA}$ & $1,47 \mathrm{aA}$ & $\begin{array}{c}1,52 \mathrm{a} \\
\mathrm{B}\end{array}$ & $\begin{array}{c}1,55 a \\
B\end{array}$ & $\begin{array}{c}1,54 \mathrm{a} \\
\mathrm{B}\end{array}$ \\
\hline SAF.1 & $\begin{array}{c}1,62 \\
\text { bA }\end{array}$ & $\begin{array}{c}1,67 \\
b B\end{array}$ & $1,58 \mathrm{aA}$ & $1,56 \mathrm{aA}$ & $1,56 \mathrm{aA}$ \\
\hline SAF.2 & $\begin{array}{c}1,60 \\
b B\end{array}$ & $\begin{array}{c}1,63 \\
b B\end{array}$ & $\begin{array}{c}1,59 a \\
B\end{array}$ & $1,54 \mathrm{aA}$ & $1,50 \mathrm{aA}$ \\
\hline SAF.3 & $\begin{array}{c}1,61 \\
b B\end{array}$ & $\begin{array}{c}1,61 \\
b B\end{array}$ & $\begin{array}{c}1,60 \mathrm{a} \\
\mathrm{B}\end{array}$ & $1,55 \mathrm{aA}$ & $1,50 \mathrm{Aa}$ \\
\hline
\end{tabular}

CV \% = 3,01 CN - Área de cerrado nativo; SAF.1 - Área com sistema cacau/banana/árvores nativas; SAF. 2 - Eucalipto/pastagem; SAF. 3 - *Eucalipto/pastagem * (conduzido a rebrota). Médias seguidas pela mesma letra não diferem estatisticamente entre si pelo teste de Scott-Knott a 5\% de probabilidade de erro.

Barbosa et al. (2017) avaliando os atributos físico-hídricos de um Cambissolo Húmico sob sistema agroflorestal no planalto Catarinense não encontrou nas camadas superficiais diferenças estatísticas entre a área nativa e os sistemas agroflorestais não estando de acordo com o presente trabalho.

Os valores das médias das variáveis, diâmetro médio ponderado e geométrico encontram-se nas Tabelas 4 e 5, respectivamente. Nas camadas superficiais $0,00-0,10$ e $0,10-0,20 \mathrm{~m}$ as áreas CN e SAF.2 apresentaram os maiores valores não diferindo estatisticamente entre si. Justifica-se isso pela intensidade de transito de máquinas para tratos culturais e colheita o que influencia diretamente na ocorrência de agregados maiores e, consequentemente maiores DMP e DMG. Na camada 0,20-0,30 m a CN possuem os maiores valores dessas variáveis diferindo estatisticamente das demais áreas. Para as camadas mais subsuperficiais $0,30-0,40$ e 0,40 $0,50 \mathrm{~m}$ as áreas em estudo não apresentaram diferenças estatísticas entre si. 
Tabela 3. Diâmetro médio ponderado do solo em áreas cultivadas com sistemas agroflorestais na região Oeste da Bahia, Barreiras, 2018.

\begin{tabular}{cccccc}
\hline Sistema & \multicolumn{5}{c}{ Camada (m) } \\
\hline & $0-0,10$ & $0,10-0,20$ & $0,20-0,30$ & $0,30-0,40$ & $0,40-0,50$ \\
CN & & $\ldots \ldots \ldots \ldots \ldots \ldots \ldots \ldots \ldots \ldots \ldots \ldots \ldots \ldots \ldots \ldots \ldots \ldots \ldots \ldots \ldots \ldots \ldots \ldots \ldots \ldots \ldots \ldots \ldots \ldots \ldots \ldots \ldots \ldots \ldots \ldots \ldots \ldots$ & \\
SAF.1 & $3,66 \mathrm{aA}$ & $5,71 \mathrm{aA}$ & $5,64 \mathrm{aA}$ & $5,56 \mathrm{aA}$ & $4,59 \mathrm{aA}$ \\
SAF.2 & $3,65 \mathrm{bA}$ & $3,96 \mathrm{bA}$ & $4,00 \mathrm{bA}$ & $4,67 \mathrm{aA}$ & $4,34 \mathrm{aA}$ \\
SAF.3 & $5,56 \mathrm{aA}$ & $4,84 \mathrm{aA}$ & $4,72 \mathrm{bA}$ & $5,06 \mathrm{aA}$ & $4,83 \mathrm{aA}$ \\
\hline
\end{tabular}

CV \%= 20,7 CN - Área de cerrado nativo; SAF.1 - Área com sistema cacau/banana/árvores nativas; SAF. 2 - Eucalipto/pastagem; SAF. 3 - *Eucalipto/pastagem * (conduzido a rebrota). Médias seguidas pela mesma letra não diferem estatisticamente entre si pelo teste de Scott- Knott a 5\% de probabilidade de erro.

Tabela 4. Diâmetro médio geométrico do solo em áreas cultivadas com sistemas agroflorestais na região Oeste da Bahia, Barreiras, 2018.

\begin{tabular}{cccccc}
\hline Sistema & \multicolumn{5}{c}{ Camada (m) } \\
\hline & $0-0,10$ & $0,10-0,20$ & $0,20-0,30$ & $0,30-0,40$ & $0,40-0,50$ \\
CN & & $\ldots \ldots \ldots \ldots \ldots \ldots \ldots \ldots \ldots \ldots \ldots \ldots \ldots \ldots \ldots \ldots \ldots \ldots \ldots \ldots \ldots \ldots \ldots \ldots \ldots \ldots \ldots \ldots \ldots \ldots \ldots \ldots \ldots \ldots \ldots \ldots \ldots \ldots \ldots \ldots$ & \\
SAF.1 & $5,34 \mathrm{aA}$ & $5,43 \mathrm{aA}$ & $5,28 \mathrm{aA}$ & $5,15 \mathrm{aA}$ & $3,71 \mathrm{aA}$ \\
SAF.2 & $2,85 \mathrm{bA}$ & $3,12 \mathrm{bA}$ & $3,28 \mathrm{bA}$ & $3,91 \mathrm{aA}$ & $3,39 \mathrm{aA}$ \\
SAF.3 & $5,15 \mathrm{aA}$ & $4,04 \mathrm{aA}$ & $3,81 \mathrm{bA}$ & $4,28 \mathrm{aA}$ & $3,97 \mathrm{aA}$ \\
\hline
\end{tabular}

CV \% = 29,91 CN - Área de cerrado nativo; SAF.1 - Área com sistema cacau/banana/árvores nativas; SAF. 2 - Eucalipto/pastagem; SAF.3 - *Eucalipto/pastagem * (conduzido a rebrota). Médias seguidas pela mesma letra não diferem estatisticamente entre si pelo teste de Scott- Knott a 5\% de probabilidade de erro.

Verifica-se na Figura 1 o percentual de agregados maiores que $2 \mathrm{~mm}$. Para a camada $0,00-0,10 \mathrm{~m}$ os maiores percentuais foram verificados nas $\mathrm{CN}$ e SAF.2. $\mathrm{Na} \mathrm{CN}$ justifica-se por ser uma área com vegetação natural e diversidade de espécie arbóreas, já no SAF.2, acredita- se que o elevado valor de agregados maiores que $2 \mathrm{~mm}$ foi provocado pela influência do sistema radicular da gramíneas que era mais abundante na camada superficial $(0,00-0,10$ $\mathrm{m})$, portanto, promovendo maior cimentação das partículas através da maior liberação de exudatos radiculares e consequentemente maior estabilidade dos agregados. $\mathrm{Na}$ camada $0,10-0,20 \mathrm{~m}$ a $\mathrm{CN}$ teve o maior percentual quando comparado com as demais áreas. Nas camadas 0,20- 0,30 e 0,30-0,40 m não foi observado diferenças estatísticas entre as áreas. 


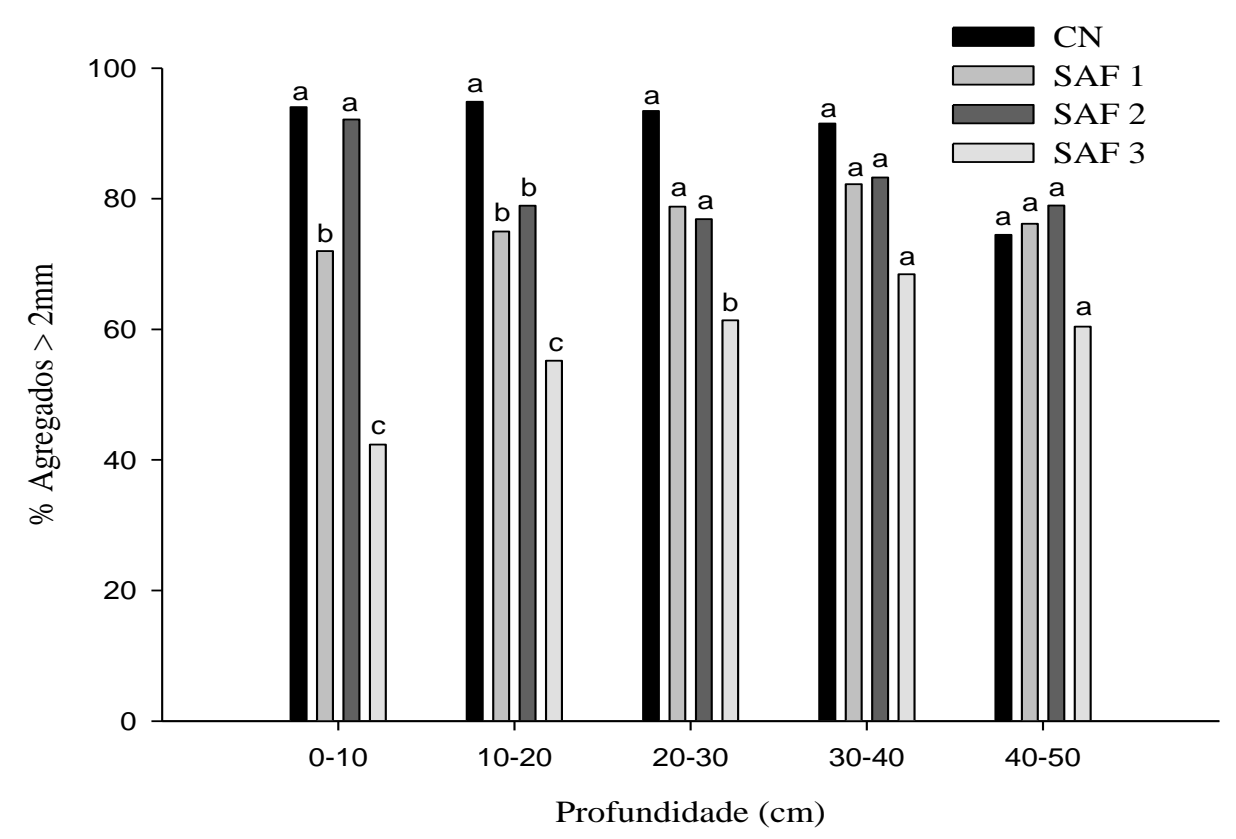

Figura 1. Percentual de agregados maiores que $2 \mathrm{~mm}$ em sistemas agroflorestais na região Oeste da Bahia, Barreiras-BA, 2018.Silva et al. (2011) avaliando atributos do solo em sistemas agroflorestais, detectaram valores de agregados maiores que $2 \mathrm{~mm}$ superior a $90 \%$ na camada superficial, tanto para área nativa como os demais sistemas, o que parte corrobora com os resultados do presente estudo.

Na Figura 2 encontram-se o percentual de agregados menores que $2 \mathrm{~mm}$. Nas camadas superficiais, 0,00-0,010 e 0,10-0,20 $\mathrm{m}$ os maiores percentuais encontram-se na SAF III, esse fato pode se justificado porque nessa área houve transito de maquinas para corte das árvores de eucalipto e isso por sua vez acarreta em uma pressão mecânica sobre essas camadas, ou seja, isso acarreta na redução dos agregados maiores estáveis em água, como também outro fator que colabora diretamente é a menor liberação de exudatos radiculares que é um fator de formação e estabilização dos agregados.

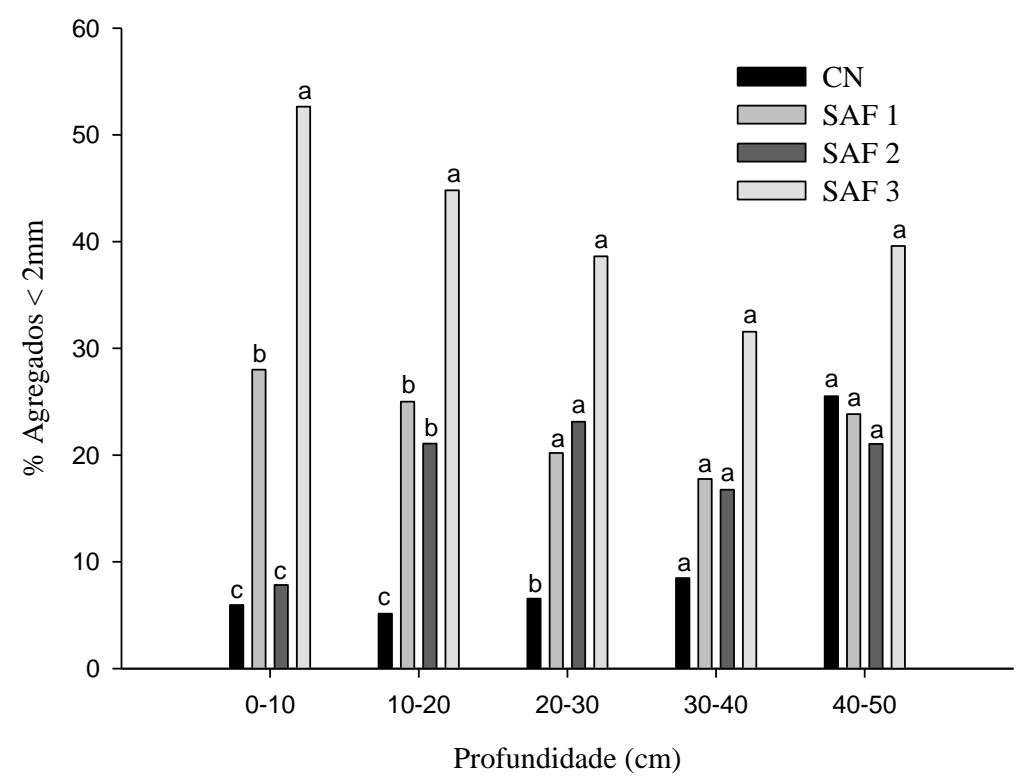

Figura 2. Percentual de agregados menores que $2 \mathrm{~mm}$ em sistemas agroflorestais na região Oeste da Bahia, Barreiras-BA, 2018. 
Revista Brasileira de Geografia Física v.12, n.07 (2019) 2660-2671.

Os estoques de carbono orgânico total encontram-se na Figura 3. Na camada 0,00$0,10 \mathrm{~m}$ os maiores estoques foram verificados na SAF.1 e SAF.3, seguido pela SAF.2. Isso ocorre nesses sistemas pela maior deposição de materiais de diferentes relações $\mathrm{C} / \mathrm{N}$, ou seja, há uma combinação das espécies arbóreas com agrícolas e isso proporciona um maior incremento nessa camada devido a maior atividade da microbiota.

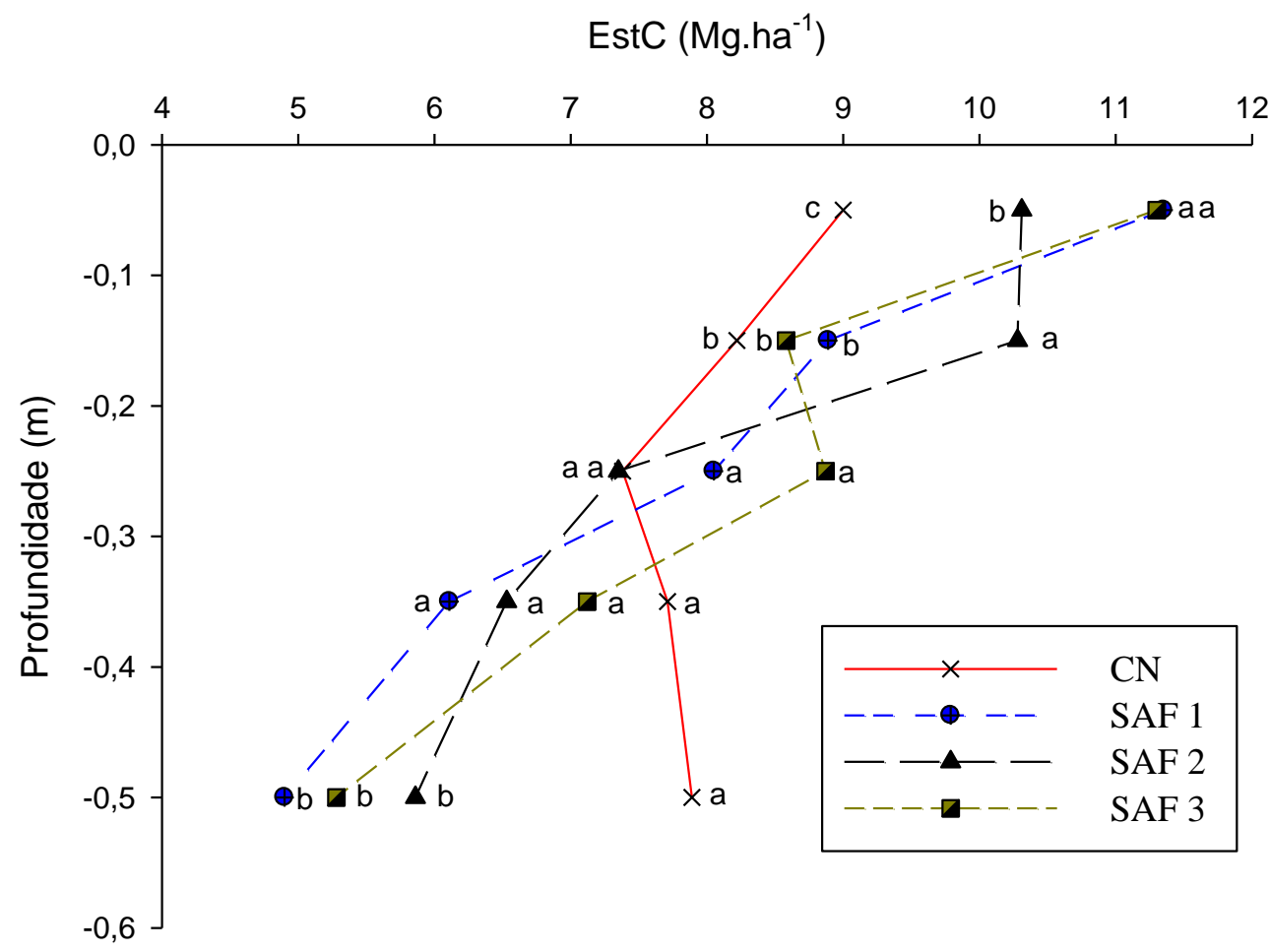

Figura 3. Estoque de carbono nas profundidades de 0,00-0,10; 0,10-0,20; 0,20-0,30; 0,30-0,40 e 0,40$0,50 \mathrm{~m}$ em sistemas agroflorestais na região Oeste da Bahia, Barreiras-BA, 2018.

Na camada $0,10-0,20 \mathrm{~m}$ os maiores estoques foram verificados na SAF.2, enquanto que nas demais áreas não foram observadas diferenças estatísticas. Esse valor também pode ter sido influenciado devido a proteção que o eucalipto oferece a gramínea e o crescimento desta que possui sistema radicular fasciculado favorece o acumulo de carbono devido a decomposição dessas raízes.

Nas camadas 0,20-0,30 e 0,30-0,40 m foi observado um comportamento semelhante para as áreas não havendo diferenças estatísticas entre as mesmas. Já na camada $0,40-0,50 \mathrm{~m}$ a área de cerrado nativo teve o maior estoque enquanto que as demais áreas não apresentaram diferenças estatísticas entre si.

Mascarenhas et al. (2017) em seus estudos com SAF'S encontraram na camada superficial valores de estoque de carbono para uma sistema agroflorestal multiestratificado inferior a área natural o que não está de acordo com o presente estudo. Já Lima et al. (2011) verificaram maiores estoques nessa camada para os sistemas agroflorestais em relação a área nativa o que corrobora com os resultados do presente estudo.

Segundo Parron et al. (2015) os estoques de carbono orgânico variam em função de diversos fatores, principalmente pelo uso e manejo do solo, ou seja, se bem manejados os SAF'S possuem grande potencial para elevar tais estoques devido sua diversidade horizontal e vertical.

Observa-se na Figura 4, o sequestro de $\mathrm{CO}_{2}$ equivalente total nas áreas estudadas na profundidade de 0,00-0,50 m. Não verificou-se diferenças estatísticas entre as áreas, ou seja, todos os sistemas de agroflorestas, independente do manejo utilizado tem potencial de sequestro de carbono no solo igual a área em equilíbrio. Sistemas surgem como alternativa para aumenta a capacidade produtiva dos solos, diminuir a pressão sobre o mesmo e ainda auxiliar de forma direta na 
retirada de $\mathrm{CO}_{2}$ da atmosfera, colaborando dessa

forma para redução do efeito estufa global.

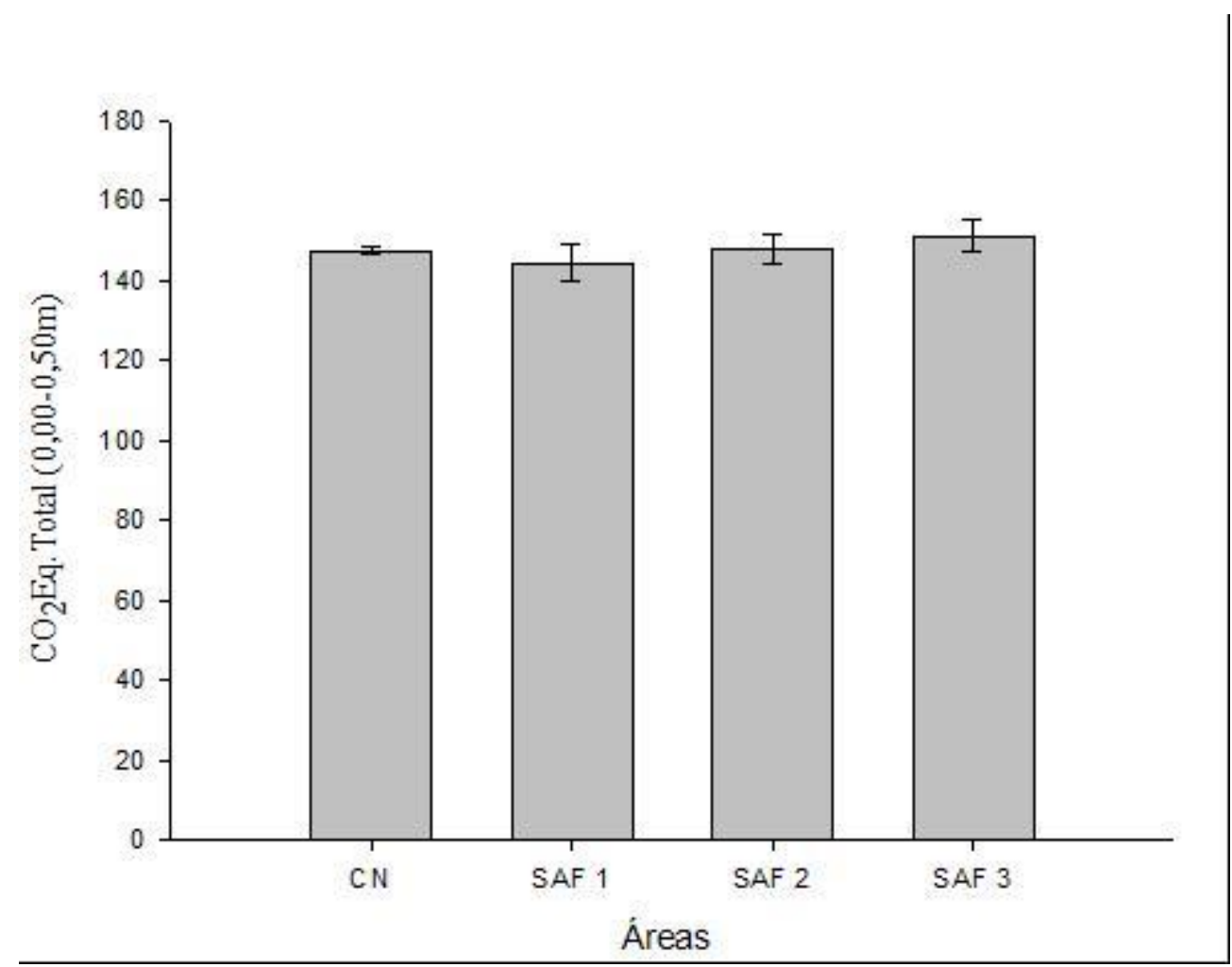

Figura 4. Sequestro de $\mathrm{CO}_{2}$ equivalente total na profundidade de 0,00-0,50 $\mathrm{m}$ em sistemas agroflorestais na região Oeste da Bahia, Barreiras-BA, 2018.

Batista (2017) em seus estudos também não encontrou diferenças estatísticas entre sistemas agroflorestais com diferentes manejos para o acumulo de carbono no solo.

\section{Análise de componentes principais}

Os resultados dos atributos dos solos sob os diferentes sistemas agroflorestais foram ordenados por meio da análise de componentes principais (ACP), em que os componentes principais 1 (CP 1) e $2(\mathrm{CP} 2)$ explicaram 65,84, 27,58\%, respectivamente, totalizando $93,42 \%$ da variabilidade total dos dados, o que de acordo com Cruz \& Regazzi (1994) e Rencher (2002) é satisfatório para avaliação por meio da dispersão gráfica dos escores em relação as primeira e segunda variáveis canônicas.

Os escores do CP 1 correlacionaram-se positivamente com a DS $(0,8017), \quad$ IEA $<2$ $\mathrm{mm}(0,8772), \mathrm{CO}(0,6878)$, Est.C $(0,7025)$ e $\mathrm{CO}_{2}$ eq $(0,7029)$ e, negativamente com DMP $(-0,9129)$, DMG $(-0,8897)$ e IEA $>2 \mathrm{~mm}(0,8772)$. Com o CP 2 a correlação positiva foi com DMP $(0,3758)$, $\operatorname{DMG}(0,4468), \mathrm{IEA}>2 \mathrm{~mm}(0,4167), \mathrm{CO}(0,7180)$, Est.C $(0,7082)$ e $\mathrm{CO}_{2}$ eq $(0,7077)$ e negativa com DS $(-0,0215)$ e IEA $<2 \mathrm{~mm}(-0,4167)$. Observa-se ainda, que $\mathrm{CO}$, Est $\mathrm{C}$ e $\mathrm{CO}_{2}$ equivalente se correlacionam entre si, bem como DMP, DMG e IEA $>2 \mathrm{~mm}$. Verifica-se também, que DMP, DMG e IEA $>2 \mathrm{~mm}$ mostraram-se com uma relação inversa ao IEA $<2 \mathrm{~mm}$ (Figura 5). 


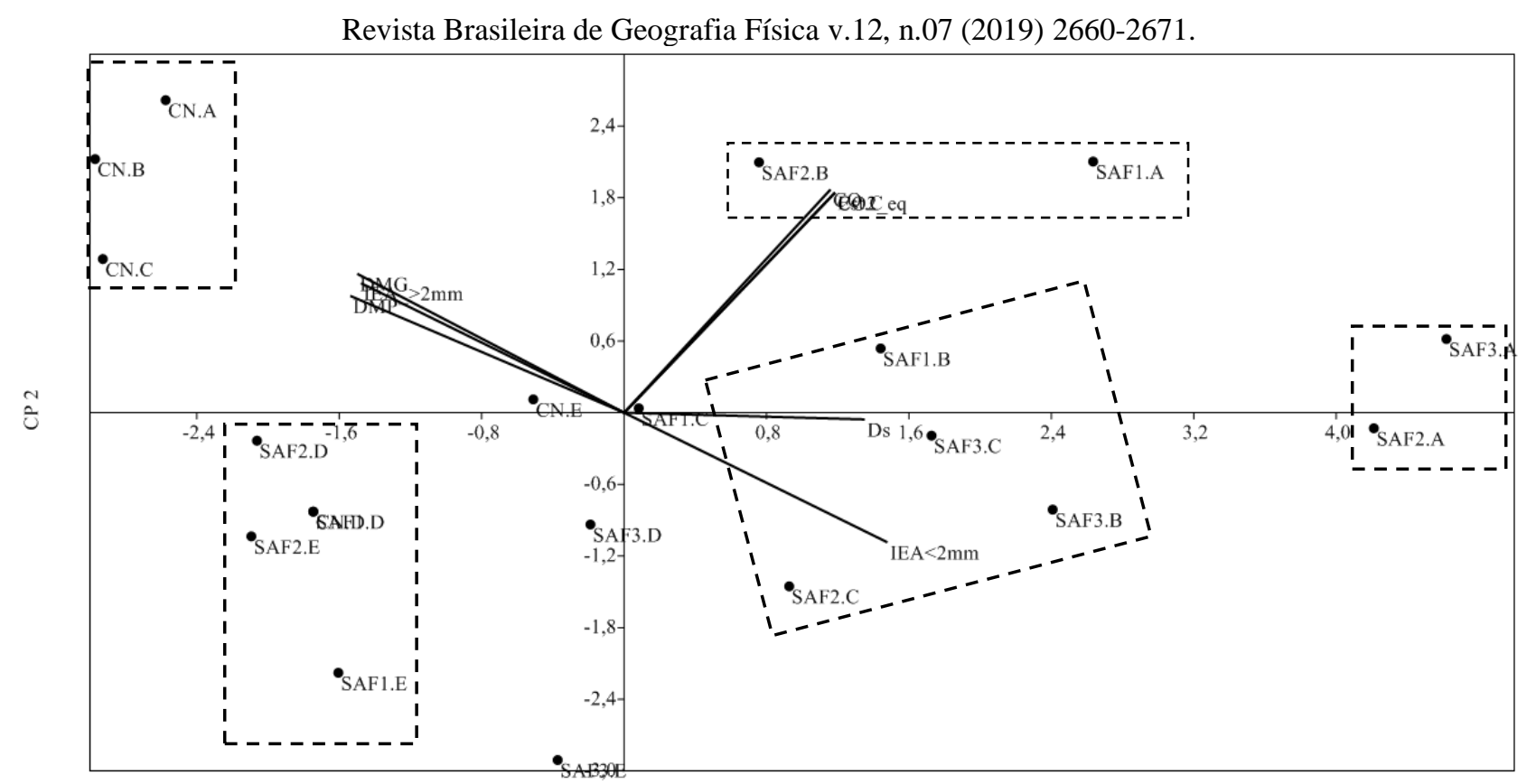

CP1

Figura 5. Componentes principais do solo sob diferentes sistemas de manejo agroflorestais. $\mathrm{CN}=$ cerrado nativo, SAFI = cacau/banana/arvore nativa, SAFII = eucalipto/pastagem, SAF III = eucalipto/pastagem com rebrota. A, B, C e D = profundidades de $0-010 \mathrm{~m} ; 0,10-0,20 \mathrm{~m} ; 0,20-0,30 \mathrm{~m} ; 0,30-0,40 \mathrm{~m}$ e $0,40-0,60 \mathrm{~m}$, respectivamente.

Ainda observa-se na Figura 5 que foi possível destacar o ambiente com $\mathrm{CN}$ nas camadas de 0 0,$10 ; 0,10-0,20$ e de $0,20-0,30 \mathrm{~m}$ formaram um grupo distinto dos demais sistemas com eucalipto (SAF.1, SAF.2 e SAF.3). Os SAF's formaram seis grupos: SAF2.E, SAF2.D, SAF1.D e SAF1.E; SAF1.B, SAF2.C, SAF3.B e SAF3.C; SAF3.D; SAF3.E; SAF2.B e SAF1.A; SAF2.A E SAF3.A. Demostrando, assim que esses sistemas florestais apresentam-se diferentes entre eles e, neles nas profundidades, fato também determinado nesses atributos quando avaliados pelo teste de média. Os atributos físicos do solo que associaram-se com o tratamento $\mathrm{CN}$ foram DMP, DMG e IEA $>2 \mathrm{~mm}$, o $\mathrm{CO}$ e $\mathrm{CO}_{2}$ eq foram responsáveis pela diferenciação do SAF.2 e SAF.1 nas profundidades de 0,10-0,20 e 0,00-0,10 m, respectivamente, enquanto que a DS associou-se com SAF.1, na camada $0,10-0,20 \mathrm{~m}$ e SAF. 3 na camada $0,20-0,30$ m. O SAF.2 (0,20-0,30 m) e SAF.3 $(0,10-0,20 \mathrm{~m})$ associaram-se ao IEA $<2 \mathrm{~mm}$ em posição oposta aos CN.A, CN.B e CN.C. Carneiro et al. (2009) em estudo semelhante, em Neossolos Quartizarênico órtico, também encontroram área de cerrado nativo formando grupo diferente das áreas com pastagem cultivada e nativa, com integração lavoura pecuária e com milho e soja em plantio direto.

\section{Conclusões}

A densidade do solo foi influenciada pelos SAF's nas camadas superficiais do solo $(0-0,10$ e 0,10-0,20 m).
O DMP e DMG, nas camadas de 0-0,10 e 0,10-0,20 m, o SAF II não se diferencia da área de cerrado nativo. O mesmo ocorre com IEA> $2 \mathrm{~mm}$ e IEA $<2 \mathrm{~mm}$.

Os sistemas agroflorestais estudados independentemente do manejo estocaram mais carbono na camada superficial $0,00-0,10 \mathrm{~m}$ que a área em equilíbrio.

Nenhum dos SAF's foi agrupado com a área de referencia nas camadas até $0,30 \mathrm{~m}$.

Apenas os SAF I e SAF II na camada de 0,30-0,40 m e SAF I e SAF II na camada de 0,40$0,50 \mathrm{~m}$ se agruparam com a área de referencia na profundidade de 0,30-040 $\mathrm{m}$.

\section{Agradecimentos}

Aos produtores rurais Jocob Lauck, Antônio Veloso e ao Eng. Florestal Moises Pedreira, por cederem suas propriedades e pelo apoio para realização desse estudo.

\section{Referências}

Almeida, A.S. et al., 2014. Biomass and carbon stocks of Sofala bay mangrove forests. Forests 5, 1967-1981.

Assis, P. C. R. et al., 2015. Atributos físicos do solo em sistemas de integração lavoura-pecuáriafloresta. Revista Brasileira de Engenharia Agrícola e Ambiental, v. 19, n. 4, p. 309-316.

Barbosa, J. dos S. 2016. Eletroquímica e carbono orgânico de um cambissolo húmico no Planalto 
Revista Brasileira de Geografia Física v.12, n.07 (2019) 2660-2671.

catarinense sob sistema agroflorestal, v. 17, n. 2, p. 49-56.

Barbosa, J.S. et al., 2017. Atributos Físico-hídricos de um Cambissolo Húmico Sob Sistema Agroflorestal no Planalto Catarinense. Floresta e Ambiente, v.24.

Campos, M. C. et al. 2016. Estoque de carbono no solo e agregados em Cambissolosob diferentes manejos no sul do Amazonas. Ambiente\&Água-An

Interdisciplinary Journal of Applied Science, v. 11, n. 2.

Cardoso, J. A., et al., 2009. Atualização da Classificação dos Solos e Elaboração de Mapas Temáticos da SubBacia Hidrográfica do Rio Grande, Bahia. In: XXXII Congresso Brasileiro de Ciência do Solo, 2009, Fortaleza - CE. O Solo e a Produção de Bioenergia: Perspectivas e Desafios.

Carvalho, J.L.N. et al, 2009.Carbon sequestration in agricultural soils in theCerrado region of the Brazil Amazon. Soiland Tillage Research v.103, p.342-34.

Coutinho, F.S., et al., 2010. Estabilidade de agregados e distribuição do carbono em Latossolo sob sistema plantio direto em Uberaba, Minas Gerais. Comunicata Scientiae 1, 100-105.

Couto, W. H. do, et al. 2016. Atributos edáficos e resistência a penetração em áreas de sistemas agroflorestais no sudoeste amazônico. Ciência Florestal, v. 26, n. 3, p. 811-823.

Cruz, C. D. e Regazzi, A. j., 1994. Modelos

biométricos aplicados ao melhoramento genético. Viçosa, MG, Universidade Federal de Viçosa, 394p.

Cunha Neto, F. V. et al. 2018. Atributos químicos e físicos do solo em áreas sob diferentes coberturas florestais e pastagem em além Paraíba - MG. Ciência Florestal, V. 28, n. 1, p. 13-34.

Embrapa. Empresa Brasileira de Pesquisa Agropecuária, 2017. Centro Nacional de Pesquisas de Solos. Manual de métodos de análises de solos. 3.ed. revista. Rio de Janeiro.

Ferreira, D.F. SISVAR versão 5.6. Disponível em: http://www.dex.ufla.br/ danielff/programas/sis var.html.

Fracetto, F. J. C. et al., 2015.Emissões de gases de efeito estufa na produção de mamona e de seus subprodutos. Revista Caatinga, v.28, n. 4, p. 9098.

Freitas, L. de. et al., 2018. Estoque de carbono de latossolos em sistemas de manejo natural e alterado. Ciência Florestal, v. 28, n. 1, p. 228239.
Hammer, Ø., Harper, D.A.T., Ryan, P.D. 2001. PAST: Paleontological statistics software package for education and data analysis. Palaeontologia Electronica4, n. 1, p. 9. Disponível em: <http://palaeoelectronica.org/2001_1/past/issue1_01.htm>

Leoni, R. C; Sampaio, N. A. de S.; Corrêa, S. M. 2017. Estatística Multivariada Aplicada ao Estudo da Qualidade do Ar. Revista Brasileira de Meteorologia, v.32, n.2, p.235-241.

Linhares, J. M. da S.et al. 2016. Variabilidade de atributos físico-químicos e dos estoques de carbono orgânico em argissolos vermelho sob sistemas agroflorestais no assentamento umari sul do amazonas. Revista Geográfica Acadêmica, v.10, n. 1, p.93-117.

Loss, A. et al. 2014.Agregação, matéria orgânica leve e carbono orgânico mineralizável em agregados do solo.Revista de laFacultad de Agronomía, La Plata (2014), v. 113, n.1, p. 1-8.

Mascarenhas, A. R. P.,et al., 2017. Atributos físicos e estoques de carbono do solo sob diferentes usos da terra em Rondônia, Amazônia Sul-Ocidental. Pesquisa florestal brasileira v.37, n. 89, 19-27.

Oliveira, C. V., 2016. Estoque de carbono em solos sob floresta nativa, sistemas agroflorestais de cacau, plantio de seringueira e pasto no Sul da Bahia. Dissertação (Mestrado) - Universidade Estadual do Sudoeste da Bahia, Programa de Pós-Graduação em Ciências Florestais, 56 f.

Parron, L. M. et al., 2015. Estoques de carbono no solo como indicador de serviços ambientais. In: Parron, L. M. et al. Serviços ambientais em sistemas agrícola e florestais do Bioma Mata Atlântica. Brasília, DF: Embrapa, p. 71-83.

Pulrolnik, K. Transformações do carbono no solo. Documentos, Embrapa Cerrados, 36p. Planaltina - DF, 2009.

Rocha Junior, P.R. et al. 2017. Soil, water, and nutrient losses from management alternatives for degraded pasture in Brazilian Atlantic Rainforest biome. Science of Total Environment. 583, p.53-63.

Rocha Junior, P. R. et al., 2018. Distribution of C and inorganic phosphorus fractions in different aggregate sizes under forestry, agroforestry system and pasture.J. Soil Sci. Plant Nutr, v.18.

Rocha, G.P. et al., 2014. Caracterização e estoques de carbono de sistemas agroflorestais no Cerrado de Minas Gerais. Ciência Rural 44.

Rossi, C. Q. et al. 2016. Vias deformação, estabilidade e características químicas de agregados em solos sob sistemas de manejo agroecológico. Pesquisa Agropecuária Brsileira, v. 51, n. 9, p. 1677-1685. 
Revista Brasileira de Geografia Física v.12, n.07 (2019) 2660-2671.

Salton, J.C. et al., 2012. Determinação da agregação do solo-Metodologia em uso na Embrapa Agropecuária Oeste. Comunicado técnico 184,Dourados-MG.

Shi. L. et al., 2018."Agroforestry systems: Metaanalysis of soil carbon stocks, sequestration processes, and future potentials." Land degradation\&development 29, 3886-3897.

Silva, A. H. et al., 2015. Atributos físicos do solo e escoamento superficial como indicadores de serviços ambientais. In: Parron, L. M. et al. Serviços ambientais em sistemas agrícola e florestais do Bioma Mata Atlântica. Brasília, DF: Embrapa, 71-83.
Silva, D. C. et al. 2011."Atributos do solo em sistemas agroflorestais, cultivo convencional e floresta nativa."Revista de Estudos Ambientais v.13, p.77-86.

Silva, S.M. 2013. Quantificação de carbono de um sistema agroflorestal em área de Cerrado no Brasil Central. Dissertação de Graduação. Faculdade UnB de Planaltina - UnB, Planaltina-DF, 2013. 46p.

Torralba, M. et al., 2016. Do European agroforestry systems enhance biodiversity and ecosystem services? A meta-analysis. Agriculture, ecosystems \& environment 230, 150-160. 